\title{
Ermakov's Superintegrable Toy and Nonlocal Symmetries
}

\author{
P.G.L. LEACH ${ }^{\dagger^{1}}$, A. KARASU $(K A L K A N L I)^{\dagger^{2}}$, M.C. NUCCI ${ }^{\dagger^{3}}$ and K. ANDRIOPOULOS ${ }^{4}$ \\ $\dagger^{1}$ School of Mathematical Sciences, Howard College, University of KwaZulu-Natal, \\ Durban 4041, Republic of South Africa \\ E-mail: leachp@ukzn.ac.za \\ $\dagger^{2}$ Department of Physics, Middle East Technical University, 06531 Ankara, Turkey \\ E-mail: akarasu@metu.edu.tr \\ $\dagger^{3}$ Dipartimento di Mathematica e Informatica, Università di Perugia, 06123 Perugia, Italy \\ E-mail: nucci@unipg.it \\ $\dagger^{4}$ Department of Information and Communication Systems Engineering, \\ University of the Aegean, Karlovassi 83 200, Greece \\ E-mail:kand@aegean.gr
}

Received September 19, 2005, in final form November 11, 2005; Published online November 15, 2005

Original article is available at http://www.emis.de/journals/SIGMA/2005/Paper018/

\begin{abstract}
We investigate the symmetry properties of a pair of Ermakov equations. The system is superintegrable and yet possesses only three Lie point symmetries with the algebra $\operatorname{sl}(2, \mathbb{R})$. The number of point symmetries is insufficient and the algebra unsuitable for the complete specification of the system. We use the method of reduction of order to reduce the nonlinear fourth-order system to a third-order system comprising a linear second-order equation and a conservation law. We obtain the representation of the complete symmetry group from this system. Four of the required symmetries are nonlocal and the algebra is the direct sum of a one-dimensional Abelian algebra with the semidirect sum of a two-dimensional solvable algebra with a two-dimensional Abelian algebra. The problem illustrates the difficulties which can arise in very elementary systems. Our treatment demonstrates the existence of possible routes to overcome these problems in a systematic fashion.
\end{abstract}

Key words: Ermakov system; reduction of order; complete symmetry group

2000 Mathematics Subject Classification: 17B80; 22E70; 34C14; 37C80

\section{Introduction}

In 1880 V. Ermakov [9] introduced a system of second-order ordinary differential equations, videlicet

$$
\begin{aligned}
& \ddot{x}+\omega^{2}(t) x=\frac{1}{x^{3}}, \\
& \ddot{y}+\omega^{2}(t) y=0,
\end{aligned}
$$

for which an invariant could be constructed by means of the operations $x \cdot(1 b)-y \cdot(1 a)$, multiplication by an integrating factor $x \dot{y}-\dot{x} y$, and integration with respect to time. The invariant obtained is

$$
I=\frac{1}{2}\left[(x \dot{y}-\dot{x} y)^{2}+\left(\frac{y}{x}\right)^{2}\right] .
$$


In the context of Ermakov's system the dependent variables $x$ and $y$ could be interpreted as spatial variables and the independent variable $t$ as time although this is not necessary. In such an interpretation the Ermakov system is a pair of coupled oscillators, one of them being nonlinear, with time-dependent frequency $\omega^{2}(t)$. The Ermakov invariant is then a generalisation of the conservation of angular momentum [23].

In another context and era the motion of a charged particle in an axially symmetric electromagnetic field was modelled in the linear approximation as the time-dependent linear oscillator

$$
\ddot{q}+\omega^{2}(t) q=0 .
$$

By an application of Kruskal's asymptotic method [19] Lewis [25, 26, 27] obtained an invariant

$$
I=\frac{1}{2}\left[(\rho p-\dot{\rho} q)^{2}+\left(\frac{q}{\rho}\right)^{2}\right]
$$

in which $\rho(t)$ is a solution of the auxiliary equation

$$
\ddot{\rho}+\omega^{2}(t) \rho=\frac{1}{\rho^{3}}
$$

and $p$ is the momentum canonically conjugate to $q$. Lewis and Riesenfeld [28] provided a quantum mechanical treatment of the time-dependent linear oscillator through the invariant (4) as a linear operator in the context of the Schrödinger Equation. This implied connection of $I$ with an Hamiltonian operator was confirmed by Leach [20] who related the Hamiltonian of the time-dependent linear oscillator

$$
H=\frac{1}{2}\left[p^{2}+\omega^{2}(t) q^{2}\right]
$$

with $I$ through a time-dependent linear generalised canonical transformation $[6,7]$

$$
Q=\frac{q}{\rho}, \quad P=\rho p-\dot{\rho} q, \quad T=\int \rho^{-2}(t) \mathrm{d} t,
$$

where $\rho(t)$ is again given by $(5)$.

The approaches of Ermakov and Lewis are different even though they embrace the same three mathematical objects. The order of introduction is different. Ermakov has a system, which could in principle be coupled although this is not found in the original treatment, and the system leads to an invariant. Lewis has a single equation and an invariant which relies upon the existence of a second equation. The modes of interpretation, the Ermakov invariant as a generalisation of the conservation of the magnitude of angular momentum and the Lewis invariant as a type of energylike Hamiltonian, reflect the differences in approach ${ }^{1}$.

A further complication had already been provided by Pinney $[46,37]$ who related the solutions of the auxiliary equation (5) to that of the time-dependent linear oscillator (3) according to

$$
\rho(t)=\left(A u^{2}+2 B u v+C v^{2}\right)^{\frac{1}{2}}
$$

where $u(t)$ and $v(t)$ are any two linearly independent solutions of (3) and the three constants $A, B$ and $C$ are related according to $A C-B^{2}=1 / W^{2}$, where $W:=u \dot{v}-\dot{u} v$ is the constant Wronskian of the basis of solutions of (3).

\footnotetext{
${ }^{1}$ In this respect the interpretation by Eliezer and Gray [8] of the Lewis invariant as a generalisation of angular momentum was rather prescient in that it preceeded by a few years the spread of knowledge of Ermakov systems beyond the region of Kiev. That Günther et al [12] could demonstrate the failure of the interpretation when the number of spatial dimensions was increased underlies the fortuitous nature of the interpretation.
} 
More recent literature $[30,33,5]$ links the solution (6) to that of the linear third-order differential equation

$$
\dddot{y}+4 \omega^{2}(t) \dot{y}+4 \omega(t) \dot{\omega}(t) y=0,
$$

where $y=\frac{1}{2} \rho^{2}$, and its integration by means of the integrating factor $y$. This connection becomes even more manifest when one recognises that (7) is the equation satisfied by the coefficient functions of the three generators of the algebra $s l(2, \mathbb{R})$ possessed by all linear $n$ th-order ordinary differential equations of maximal symmetry ${ }^{2}$ and that $s l(2, \mathbb{R})$ is also the algebra of the Lie point symmetries of the Ermakov-Pinney equation (1a), (5).

Around 1978 the systems of Ermakov became known in the West - there is more than a little folklore about the mode of dissemination - and immediately attracted a great deal of attention; for a modest sampling see [47, 48, 49]. The flood of papers did diminish somewhat, but more recent articles $[13,14,15]$ indicate that the mathematical and physical riches of Ermakov systems have not been exhausted.

In this paper we wish to treat a two-dimensional Ermakov system in a manner analogous to the treatment of the 'beloved' equation ${ }^{3}$

$$
\dddot{x}+3 x \dot{x}+x^{3}=0
$$

by Karasu et al [16] in which a doubled version of (8) was studied from the viewpoint of its symmetries and integrability in the sense of Painlevé. The collapse of the Lie point symmetry algebra from the single (8) to its dual was dramatic. In that treatment there existed a linearising transformation to a pair of third-order ordinary differential equations and the Lie point symmetries of the third-order equations enabled the complete symmetry group of the system of second-order equations to be obtained in terms of a set of five nonlocal symmetries which was distinguished by being Abelian.

The complete symmetry group of a system of ordinary differential equations is the group of the algebra of Lie symmetries required to specify the system completely. The concept was introduced by Krause [17, 18] in a study of the Kepler Problem. The absence of a sufficient number of Lie point symmetries for the purpose caused Krause to introduce nonlocal symmetries by an ingenious strategy which has proved to be useful elsewhere [41]. The necessity for the introduction of an Ansatz for the structure of nonlocal symmetries was successfully challenged by Nucci [38] who used the method of reduction of order [39] to obtain the requisite number of symmetries wholly through point symmetries - naturally with a coupling to a nonlocal transformation in the case of those symmetries which were nonlocal in the original representation. This same method has been found to apply to a wide variety of systems [40, 42] and has revealed an unanticipated algebraic identity of somewhat dissimilar problems. The present theoretical development of the properties of complete symmetry groups can be found in $[1,2,3,4]$.

\footnotetext{
${ }^{2}$ There are some variations in the precise forms of the coefficients of $\dot{y}$ and $y$ to allow for the value of $n$; see Mahomed et al [30]. Indeed the third-order equation (7) as adjusted for the order of the equation under study, provides a unifying feature to the study of the Lie algebras of $n$ th-order ordinary differential equations as it is always present for equations of maximal symmetry. For $n \geq 3$ the other symmetries come from the solution of an $n$ th-order differential equation and a first-order differential equation. The solutions of the $n$ th-order equation and third-order equation are related to the solutions of a second-order equation of maximal symmetry [32]. In fact this relationship leads to a simple demonstration of Pinney's solution (6). For $n=2$ another second-order differential equation intrudes to provide the noncartan symmetries. This second-order differential equation is the adjoint of the second-order equation under study [50].

${ }^{3}$ 'L'équation adoré' as (8) was dubbed, perhaps a little irreverently, by the recently and sadly late Mark Feix in 1996 after yet another attractive property of this remarkable equation was revealed in the work of his student, Claude Géronimi.
} 
The system which we treat is

$$
\begin{aligned}
& \ddot{x}=\frac{1}{x^{3}}, \\
& \ddot{y}=\frac{1}{y^{3}}
\end{aligned}
$$

in which each component is the simplest form of the Ermakov-Pinney equation possible and no coupling between the two equations exists. The reader sceptical of the potential difference between a single Ermakov-Pinney equation and a pair cloned as above is referred to the system discussed by Karasu et al [16] in which these differences are displayed in detail. We keep the system at its simplest to avoid masking the clarity of the concept under consideration behind a complexity of model ${ }^{4}$. We note that (9) is related to the more usual form of Ermakov systems by means of a point transformation [23].

We examine the system (9) from the point of view of its Lie point symmetries and find that these are insufficient to specify completely the system. A recent work of Nucci et al [44] on the complete symmetry group of (9a) does provide an easy route to the complete symmetry group of the system (9) although the ease does follow from the prior passage of the route. For a single Ermakov-Pinney equation that route required the application of the method of reduction of order. The method relies upon the existence of an ignorable coordinate. In the case of (9) time is the obvious ignorable coordinate. The choice of a new independent coordinate is not obvious ${ }^{5}$. However, a transition to polar coordinates makes the choice traditionally obvious. In polar coordinates the method of reduction of order leads to the combination of a linear second-order ordinary differential equation and a trivial first-order differential equation, i.e. a conservation law, reminiscent of the reduction of the MIC-Z Problem [24] in addition to problems more obviously connected to the Kepler Problem.

The reduction provides a source of Lie point symmetries which can then be translated to the original coordinates and equations to give a suite of symmetries from which the complete symmetry group of system (9) can be determined. Fortunately the theorems developed by Andriopoulos et al [4] obviate the necessity for lengthy calculations involving nonlocal symmetries for we can use the symmetries of the complete symmetry group of the derivate system and the transitive properties of complete symmetry groups to arrive at the complete symmetry group of (9). We note that in this derivation the nonuniqueness of the representation given by Nucci et al is replicated due to the known property of the possession by linear second-order ordinary differential equations of at least three representations of their complete symmetry groups [22, 2].

The interesting feature of system (9) is that it is rather light on Lie point symmetries and yet, as we see below, is a superintegrable system in the standard sense in that it possesses three first integrals.

The essential points of this paper fall into two parts. Individually (9a) and (9b) are endowed with sufficient symmetry to ensure an easy integration. In this context we mean sufficient Lie point symmetries as is the standard for second-order equations and usual for higher order equations. The simple cojoining of the two equations produces the situation in which the number of Lie point symmetries is formally fewer than that required for easy integration and yet the ease of integration remains. We use this simple system to emphasise the problems of moving from a scalar equation to a system of equations. The second part of the paper deals with the solution of the problem posed by the lack of Lie point symmetries by comparison with the individual scalar second-order equations. The resolution of such problems is not necessarily by means of

\footnotetext{
${ }^{4}$ In a sense this already happened with complete symmetry groups which entailed the use of nonlocal symmetries $[17,18]$ whereas the idea can be displayed in the context of point symmetries and the most elementary of ordinary differential equations $[1,2,3]$.

${ }^{5}$ In the case of just (9a) this is obviously $x$.
} 
a recipe. Were this so, the solution of systems of nonlinear equations would be no more difficult than that of scalar equations. Anyone with the slightest acquaintance with the problem of the solution of systems of nonlinear equations knows the foolishness of that position. What we do in this paper reflects the two parts. Firstly we delineate the problems encountered in the simple act of cojoining two integrable equations as far as the usual criteria of integrability in the Lie theory are concerned. Secondly we demonstrate how those problems are overcome. The particular resolution of these problems may be unique to the system, but the techniques are of a general nature and belong to the standard repertoire to be used when a system displays refractory qualities. The techniques used here were not designed for this system. They exist and may be employed generally. The success of their employment depends upon the specific nature of the system under consideration.

We emphasise that the system we study (9) is quite artificial and is studied more to illustrate properties and methods than to provide novel application. Yet we find it an appropriate object for our attentions in this 125th anniversary of its genesis in this noble city of Kiev by one of its now well-known sons.

\section{The superintegrable toy}

There are three Lie point symmetries of system (9), videlicet

$$
\begin{aligned}
& \Gamma_{1}=\partial_{t}, \\
& \Gamma_{2}=t \partial_{t}+\frac{1}{2}\left(x \partial_{x}+y \partial_{y}\right), \\
& \Gamma_{3}=t^{2} \partial_{t}+t\left(x \partial_{x}+y \partial_{y}\right),
\end{aligned}
$$

with the Lie Brackets

$$
\left[\Gamma_{1}, \Gamma_{2}\right]=\Gamma_{1}, \quad\left[\Gamma_{1}, \Gamma_{3}\right]=2 \Gamma_{2}, \quad\left[\Gamma_{2}, \Gamma_{3}\right]=\Gamma_{3}
$$

characteristic of the algebra $s l(2, \mathbb{R})$. The system (9) may be regarded as a pair of first integrals consequent upon the use of the integrating factors $\rho$ and $\sigma$, respectively, of the system

$$
\begin{aligned}
& \dddot{\rho}=0, \\
& \dddot{\sigma}=0,
\end{aligned} \Longrightarrow \begin{aligned}
& \rho \dddot{\rho}=0, \\
& \sigma \dddot{\sigma}=0,
\end{aligned} \Longrightarrow \begin{aligned}
& \rho \ddot{\rho}-\frac{1}{2} \dot{\rho}^{2}=\frac{1}{2} A, \\
& \sigma \ddot{\sigma}-\frac{1}{2} \dot{\sigma}^{2}=\frac{1}{2} B,
\end{aligned} \Longrightarrow \begin{aligned}
& \rho=\frac{1}{2} x^{2}, \\
& \sigma=\frac{1}{2} y^{2},
\end{aligned} \Longrightarrow \begin{aligned}
& x^{3} \ddot{x}=A, \\
& y^{3} \ddot{y}=B
\end{aligned}
$$

with the specific choice of the constants of integration $A$ and $B$ being unity. The third-order system is of maximal symmetry with

$$
\begin{array}{ll}
\Lambda_{11}=\partial_{\rho}, & \Lambda_{21}=\partial_{\sigma}, \\
\Lambda_{12}=t \partial_{\rho}, & \Lambda_{22}=t \partial_{\sigma}, \\
\Lambda_{13}=\frac{1}{2} t^{2} \partial_{\rho}, & \Lambda_{23}=\frac{1}{2} t^{2} \partial_{\sigma}, \\
\Lambda_{14}=\rho \partial_{\rho}, & \Lambda_{24}=\sigma \partial_{\sigma}, \\
\Lambda_{15}=\sigma \partial_{\rho}, & \Lambda_{25}=\rho \partial_{\sigma}, \\
& \\
\Lambda_{c 1}=\partial_{t}, & \\
\Lambda_{c 2}=t \partial_{t}, &
\end{array}
$$

and only three of these survive in the integrals whether they be treated as functions or equations ${ }^{6}$.

\footnotetext{
${ }^{6}$ System (11) has the usual ambiguity of self-similar symmetries for linear systems of maximal symmetry. The nonlinearity of (9) enforces the precise form of $\Gamma_{2}$ in (10). In terms of the variables $\rho$ and $\sigma$ the symmetries $\Gamma_{2}$ and $\Gamma_{3}$ are in the standard form for the representation of $s l(2, \mathbb{R})$ appropriate to equations of the third order. The transformation to $x$ and $y$ gives the standard form for second-order equations [30], but we do well to recall that the symmetries are from the third-order system (11) as listed in (12).
} 
Two first integrals of system (9) are obviously

$$
I=\frac{1}{2}\left(\dot{x}^{2}+\frac{1}{x^{2}}\right) \quad \text { and } \quad J=\frac{1}{2}\left(\dot{y}^{2}+\frac{1}{y^{2}}\right) .
$$

The third first integral is the Ermakov invariant

$$
K=\frac{1}{2}\left[(x \dot{y}-\dot{x} y)^{2}+\frac{x^{2}}{y^{2}}+\frac{y^{2}}{x^{2}}\right]
$$

which is constructed in the same manner as for the Ermakov invariant (2) of system (1). System (9) is of the fourth order and has three autonomous constants of the motion. Hence system (9) is superintegrable.

The autonomous integrals can all be derived from the symmetry $\partial_{t}$ if one uses Lie's method (cf. [21]). Invariance under $\Gamma_{1}$ means that the integral is autonomous, i.e. of the form $F(x, y, \dot{x}, \dot{y})$. The associated Lagrange's system for $\mathrm{d} F / \mathrm{d} t=0$ is

$$
\frac{\mathrm{d} x}{\dot{x}}=\frac{\mathrm{d} y}{\dot{y}}=\frac{\mathrm{d} \dot{x}}{x^{-3}}=\frac{\mathrm{d} \dot{y}}{y^{-3}}
$$

when (9) are taken into account. The combination of the first and third of (14) leads to $I$ and of the second and fourth to $J$. The determination of $K$ is a little more complicated. The combinations $\dot{y} \cdot(14 \mathrm{a})-\dot{x} \cdot(14 \mathrm{c})+y \cdot(14 \mathrm{~b})-x \cdot(14 \mathrm{~d})$ and $y \cdot(14 \mathrm{a})-x \cdot(14 \mathrm{~b})$ give

$$
\frac{\mathrm{d}(x \dot{y}-\dot{x} y)}{\frac{x}{y^{3}}-\frac{y}{x^{3}}}=\frac{y \mathrm{~d} x-x \mathrm{~d} y}{y \dot{x}-x \dot{y}}
$$

from which the third integral $K$ follows.

Although $\Gamma_{1}$ generates the three autonomous first integrals of system (9), each integral possesses an infinite number of Lie point symmetries. In the case of $K$ these are [10, 11]

$$
\Sigma_{K}=f(t) \partial_{t}+\frac{1}{2} \dot{f}(t)\left(x \partial_{x}+y \partial_{y}\right),
$$

where $f(t)$ is an arbitrary, differentiable, function. In the cases of $I$ and $J$ the infinite classes of Lie point symmetries are respectively

$$
\begin{aligned}
& \Sigma_{I}=\partial_{t}+f(t, x, y) \partial_{y}, \\
& \Sigma_{J}=\partial_{t}+f(t, x, y) \partial_{x}
\end{aligned}
$$

which are both perhaps to be expected and not as interesting as in the case of $K$.

As an amusing endpoint to this discussion of the integrals we note that the integrals associated with $\Gamma_{3}$ are

$$
I_{3}=\frac{1}{2}\left\{(t \dot{x}-x)^{2}+\left(\frac{t}{x}\right)^{2}\right\}, \quad J_{3}=\frac{1}{2}\left\{(t \dot{y}-y)^{2}+\left(\frac{t}{y}\right)^{2}\right\}
$$

and $K$ as given in (13). If one uses the point transformation

$$
T=-\frac{1}{t}, \quad X=\frac{x}{t}, \quad Y=\frac{y}{t},
$$

the integrals $I$ and $J$ are recovered. (Obviously $K$ is invariant under the point transformation.) The transformation (15) is a simple instance of the generalised canonical transformation $[6,7]$

$$
T=\int \rho^{-2}(t) \mathrm{d} t, \quad Q=\frac{q}{\rho}, \quad P=\rho p-\dot{\rho} q
$$

which has found much application in both Hamiltonian and Quantum Mechanics [20, 29, 45] .

\footnotetext{
${ }^{7}$ Apart from $K$ the symmetry $\Gamma_{2}$ has the two integrals $I_{2}=\dot{x}(t \dot{x}-x)+t / x^{2}$ and $J_{2}=\dot{y}(t \dot{y}-y)+t / y^{2}$. This
} 


\section{Complete symmetry group}

The Lie algebra $\operatorname{sl}(2, \mathbb{R})$ is not found as a constituent of a complete symmetry group since the Lie Brackets show a redundancy of operators and this thereby leads to a violation of the condition of minimality which is one of the requirements for a representation of a complete symmetry group [1,44]. This then poses the problem of finding the elements of the algebra of the complete symmetry group for (9) since the only Lie point symmetries are the elements of $s l(2, \mathbb{R})$. Karasu et al [16], when confronted with a similar obstacle to progress in determination, made use of a nonlocal transformation to a third-order system of the form of (11) and were able to use the nonlocal forms of the remainder of (12) at the second-order level to express the complete symmetry group of the system in terms of a five-dimensional Abelian algebra of nonlocal symmetries.

That option is not so apparent for the present system. However, we may make use of an extension of the work of Nucci et al [44] to obtain indirectly a representation of the complete symmetry group for the system (9). They, by a complex sequence of analyses and transformations facilitated by some recent theorems of Andriopoulos et al [4], were able to represent the complete symmetry group of a single Ermakov-Pinney equation. In the notation adopted here, (9a) say, is completely described by the three symmetries

$$
\Delta_{1}=\partial_{t}, \quad \Delta_{2}=\left(\int \frac{\mathrm{d} t}{\dot{x}^{2}}\right) \partial_{t}, \quad \Delta_{3}=\frac{1}{2}\left(\int\left(1+\frac{1}{x^{2} \dot{x}^{2}}\right) \mathrm{d} t\right) \partial_{t} .
$$

It is a simple matter to infer that the system (9) is completely specified by (16) plus

$$
\Delta_{4}=\left(\int \frac{\mathrm{d} t}{\dot{y}^{2}}\right) \partial_{t}, \quad \Delta_{5}=\frac{1}{2}\left(\int\left(1+\frac{1}{y^{2} \dot{y}^{2}}\right) \mathrm{d} t\right) \partial_{t}
$$

to give the five symmetries expected by the general theory [2].

We verify that $\Delta_{1}-\Delta_{5}$ are a valid representation of the complete symmetry group by considering the actions of $\Delta_{1}^{[2]}-\Delta_{5}^{[2]}$ on the general two-dimensional system

$$
\begin{aligned}
& \ddot{x}=f(t, x, y, \dot{x}, \dot{y}), \\
& \ddot{y}=g(t, x, y, \dot{x}, \dot{y}) .
\end{aligned}
$$

The action of $\Delta_{1}$ removes $t$ from both of $f$ and $g$. The second extensions of $\Delta_{2}$ and $\Delta_{4}$ are

$$
\Delta_{2}^{[2]}=\left(\int \frac{1}{\dot{x}^{2}} \mathrm{~d} t\right) \partial_{t}-\frac{1}{\dot{x}} \partial_{\dot{x}} \quad \text { and } \quad \Delta_{4}^{[2]}=\left(\int \frac{1}{\dot{y}^{2}} \mathrm{~d} t\right) \partial_{t}-\frac{1}{\dot{y}} \partial_{\dot{y}} .
$$

They remove $\dot{x}$ and $\dot{y}$ from $f$ and $g$. The relevant parts of the second extensions of $\Delta_{3}$ and $\Delta_{5}$ are

$$
\Delta_{3}^{[2]}=\left\{-f+\frac{1}{x^{3}}\right\} \partial_{\ddot{x}} \quad \text { and } \quad \Delta_{5}^{[2]}=\left\{-g+\frac{1}{y^{3}}\right\} \partial_{\ddot{y}}
$$

from which the recovery of (9) is evident.

in itself is interesting as the three integrals $I_{1}, I_{2}$ and $I_{3}$, respectively $J_{1}, J_{2}$ and $J_{3}$, are generalisations of the quadratic integrals of the one-dimensional free particle which for $\ddot{x}=0$ are $\frac{1}{2} \dot{x}^{2}, \dot{x}(t \dot{x}-x)$ and $\frac{1}{2}(t \dot{x}-x)^{2}$. These integrals are obviously products of the two linear integrals $\dot{x}$ and $t \dot{x}-x$. The nonlinear Ermakov system does not possess linear integrals. However, the quadratic integrals could be considered as the inner products of the nonconserved vectors $\boldsymbol{i}_{x}=\dot{x} \hat{\boldsymbol{a}}_{1}+\frac{1}{x} \hat{\boldsymbol{a}}_{2}, \boldsymbol{i}_{y}=\dot{y} \hat{\boldsymbol{e}}_{1}+\frac{1}{y} \hat{\boldsymbol{e}}_{2}, \boldsymbol{j}_{x}=(t \dot{x}-x) \hat{\boldsymbol{a}}_{1}+\frac{t}{x} \hat{\boldsymbol{a}}_{2}$ and $\boldsymbol{j}_{y}=(t \dot{y}-y) \hat{\boldsymbol{e}}_{1}+\frac{t}{y} \hat{\boldsymbol{e}}_{2}$, where $\hat{\boldsymbol{a}}_{i}$ and $\hat{\boldsymbol{e}}_{i}, i=1,2$, are unit vectors. In further contrast to the free particle the inner products of the $x$ vectors and the $y$ vectors are not conserved. 


\section{The method of reduction of order}

The method of reduction of order was used very effectively by Nucci [38] to obtain the complete symmetry group for the Kepler Problem, the representation of which is mainly in terms of nonlocal symmetries, in terms of Lie point symmetries of the reduced system. The reader is referred to Nucci et al [39] for a detailed description of the method. Essentially the differential equations are written as a system of first-order ordinary differential equations and the order of the system is reduced by the removal of ignorable variables. Eventually one (or more) variable(s) is (are) eliminated to give at least one equation of the second order so that Lie's algorithm for the determination of Lie point symmetries can be implemented as a finite procedure. The symmetries obtained are then translated back to symmetries of the original system. The identification of the complete symmetry group is facilitated by the theorems on transitivity recently obtained by Andriopoulos et al [4].

The Ermakov System as presented in (9) is not satisfactory for the application of the method of reduction of order since there is no preferred new variable after the time, as an ignorable coordinate, is eliminated. However, conversion of the system to a representation in terms of plane polar coordinates through $x=r \cos \theta$ and $y=r \sin \theta$ and recombination into radial and tangental components, videlicet

$$
\begin{aligned}
& \ddot{r}-r \dot{\theta}^{2}-r^{-3}(\tan \theta+\cot \theta)^{2}=0, \\
& r \ddot{\theta}+2 \dot{r} \dot{\theta}+\frac{1}{2} r^{-3}(\tan \theta+\cot \theta)^{\prime}=0,
\end{aligned}
$$

where the prime denotes differentiation with respect to $\theta$, does provide a good basis for the reduction since ultimately one can envisage a polar representation of the orbit.

We define the variables $w_{1}=r, w_{2}=\theta, w_{3}=\dot{r}$ and $w_{4}=\dot{\theta}$ so that the system (18) can be written in terms of the four first-order ordinary differential equations

$$
\begin{aligned}
& \dot{w}_{1}=w_{3}, \\
& \dot{w}_{2}=w_{4}, \\
& \dot{w}_{3}=w_{1} w_{4}^{2}+\frac{a\left(w_{2}\right)}{w_{1}^{3}}, \\
& \dot{w}_{4}=-2 \frac{w_{3} w_{4}}{w_{1}}-\frac{a^{\prime}\left(w_{2}\right)}{2 w_{1}^{4}},
\end{aligned}
$$

where we write $(\tan \theta+\cot \theta)^{2}=a(\theta)$ to keep a certain compactness of notation. Obviously the system is autonomous, i.e. $t$ is an ignorable variable.

An obvious candidate as a new independent variable is $w_{2}$ which we write as $y$. The ratios of (19a), (19c) and (19d) with (19b) give the reduced third-order system

$$
\begin{aligned}
\frac{\mathrm{d} w_{1}}{\mathrm{~d} y} & =\frac{w_{3}}{w_{4}}, \\
\frac{\mathrm{d} w_{3}}{\mathrm{~d} y} & =w_{1} w_{4}+\frac{a(y)}{w_{1}^{3} w_{4}}, \\
\frac{\mathrm{d} w_{4}}{\mathrm{~d} y} & =-2 \frac{w_{3}}{w_{1}}-\frac{a^{\prime}(y)}{2 w_{1}^{4} w_{4}} .
\end{aligned}
$$

The next step is to introduce a second-order differential equation into the system so that Lie's algorithm becomes finite. The obvious variable for elimination is $w_{3}$ for from (20a) we have the simple relationship $w_{3}=w_{4} w_{1}^{\prime}$, where now the prime refers to $y$. 
The system of second-order differential equation and first-order differential equation is

$$
\begin{aligned}
& w_{4} w_{1}^{\prime \prime}+w_{4}^{\prime} w_{1}^{\prime}=w_{1} w_{4}+\frac{a(y)}{w_{1}^{3} w_{4}}, \\
& w_{4}^{\prime}=-2 \frac{w_{4} w_{1}^{\prime}}{w_{1}}-\frac{a^{\prime}(y)}{2 w_{1}^{4} w_{4}} .
\end{aligned}
$$

At this stage one can apply Lie's algorithm, but some æsthetic reshaping of system (21) is informative. Equation (21b) can be written in the explicitly conservative form

$$
u_{2}^{\prime}=0
$$

where

$$
u_{2}:=w_{1}^{4} w_{4}^{2}+a(y) .
$$

We recognise $u_{2}(y)$ as related to the Ermakov Invariant appropriate to system (9), but we must recall, as the explicit presence of the independent variable reminds us, that this is in terms of the independent variable $y$ and not the original independent variable $t$. The distinction is technical since $\mathrm{d} u_{2} / \mathrm{d} t=u_{2}^{\prime} \dot{y}$.

We introduce the new dependent variable into (21a) and after some manipulation arrive at

$$
\left(u_{2}-a\right) u_{1}^{\prime}-\frac{1}{2} a^{\prime} u_{1}^{\prime}+u_{2} u_{1}=0
$$

where we have introduced the new dependent variable $u_{1}=1 / w_{1}$.

We have reduced the original fourth-order system to a third-order system comprising one second-order equation (23) and one first-order equation (22). The first-order equation has the nature of a conservation law. We recall that the same type of reduction has been demonstrated for the Kepler Problem, problems based upon it and the MIC-Z system. The question which naturally arises is one of the equivalence of these somewhat disparate systems. The resolution of the question is found in consideration of (23). As a linear equation this can be reduced to that of the simple harmonic oscillator - the form of the second-order equation taken in the case of the reduction of the Kepler Problem - by means of a Kummer-Liouville transformation. The transformation of (23) to a nonautonomous oscillator requires simply a change in the dependent variable. However, to go from the nonautonomous oscillator to the simple harmonic oscillator requires a redefinition of the independent variable. In the usual area of application, i.e. the treatment of the time-dependent oscillator, the introduction of 'new time' is simply a rescaling

of a variable without bound. Here the independent variable has a geometrical meaning and a rescaling of the polar angle changes the geometry. Consequently we must conclude that there is an essential difference between the two classes of problem despite the formal equivalence obtained under the procedure of the method of reduction of order.

\section{Symmetries of the original system and its complete symmetry group}

The Lie point symmetries of the system (23), (22) are given in composite form as

$$
\Gamma=\left(\alpha+\beta u_{1}\right) \partial_{y}+\left(\sigma u_{1}^{2}+\gamma u_{1}+\delta\right) \partial_{u_{1}}+\epsilon \partial_{u_{2}},
$$

where

$$
\begin{aligned}
& \sigma=\frac{\partial \beta}{\partial y}+\frac{a^{\prime}}{2\left(u_{2}-a\right)} \beta \\
& \gamma=\gamma_{0}+\frac{1}{2}\left(\frac{\partial \alpha}{\partial y}+\frac{\alpha a^{\prime}-\epsilon}{u_{2}-a}\right)
\end{aligned}
$$


$\gamma_{0}$ is a constant, $\epsilon$ is an arbitrary function of $u_{2}$ and $\alpha, \beta$ and $\delta$ solutions of the equations

$$
\begin{aligned}
\frac{\partial \alpha}{\partial y}+ & {\left[\frac{4 u_{2}}{u_{2}-a}+\frac{\partial}{\partial y}\left(\frac{a^{\prime}}{u_{2}-a}\right)^{\prime}-\frac{1}{4}\left(\frac{a^{\prime}}{u_{2}-a}\right)^{2}\right] \frac{\partial \alpha}{\partial y} } \\
& +\frac{1}{2} \frac{\partial}{\partial y}\left[\frac{4 u_{2}}{u_{2}-a}+\frac{\partial}{\partial y}\left(\frac{a^{\prime}}{u_{2}-a}\right)-\frac{1}{4}\left(\frac{a^{\prime}}{u_{2}-a}\right)^{2}\right]^{\prime} \alpha \\
& =\frac{1}{2\left(u_{2}-a\right)}\left\{\frac{\partial}{\partial y}\left(\frac{a^{\prime}}{u_{2}-a}\right)+\frac{4 a}{u_{2}-a}\right\}, \\
\frac{\partial \beta}{\partial y}+ & \frac{1}{2} \frac{a^{\prime}}{u_{2}-a} \frac{\partial \beta}{\partial y}+\frac{1}{2}\left[\frac{2 u_{2}}{u_{2}-a}+\left(\frac{a^{\prime}}{u_{2}-a}\right)^{\prime}\right] \beta=0, \\
\frac{\partial \delta}{\partial y}- & \frac{1}{2} \frac{a^{\prime}}{u_{2}-a} \frac{\partial \delta}{\partial y}+\frac{u_{2}}{u_{2}-a} \delta=0 .
\end{aligned}
$$

We note that the equation for $\beta$ is the adjoint of the equation for $\delta$.

Two of the relevant symmetries are [2] the two solution symmetries of (23) given by the $\delta$ equation. We write these as

$$
\Sigma_{1}=s_{1} \partial_{u_{1}}, \quad \Sigma_{2}=s_{2} \partial_{u_{1}},
$$

where $s_{1}$ and $s_{2}$ are two linearly independent solutions of the $\delta$ equation.

We firstly examine the effects of these two symmetries. The general pair of second-order equation and first-order equation is

$$
\begin{aligned}
& u_{1}^{\prime \prime}=f\left(y, u_{1}, u_{2}, u_{1}^{\prime}\right), \\
& u_{2}^{\prime}=g\left(y, u_{1}, u_{2}, u_{1}^{\prime}\right) .
\end{aligned}
$$

The application of $\Sigma_{1}^{[2]}$ gives

$$
\begin{aligned}
& s_{1}^{\prime \prime}=\frac{\partial f}{\partial u_{1}}+s_{1}^{\prime} \frac{\partial f}{\partial u_{1}^{\prime}} \\
& 0=s_{1} \frac{\partial g}{\partial u_{1}}+s_{1}^{\prime} \frac{\partial g}{\partial u_{1}^{\prime}}
\end{aligned}
$$

from the solutions of which the two equations in (27) now become

$$
\begin{aligned}
& u_{1}^{\prime \prime}=\frac{s_{1}^{\prime \prime}}{s_{1}}+F\left(y, s_{1} u_{1}^{\prime}-s_{1}^{\prime} u_{1}^{\prime}, u_{2}\right), \\
& u_{2}^{\prime}=G\left(y, s_{1} u_{1}^{\prime}-s_{1} u_{1}^{\prime}, u_{2}\right),
\end{aligned}
$$

where $F$ and $G$ are arbitrary functions of the indicated arguments.

After the application of $\Sigma_{2}^{[2]}$ to (28) we have

$$
\begin{aligned}
u_{1}^{\prime \prime} & =\frac{s_{1}^{\prime \prime}}{s_{1}} u_{1}+\frac{1}{s_{1}} \frac{s_{1} s_{2}^{\prime \prime}-s_{1}^{\prime \prime} s_{2}}{s_{1} s_{2}^{\prime}-s_{1}^{\prime} s_{2}}\left(s_{1} u_{1}^{\prime}-s_{1}^{\prime} u_{1}\right)+\mathcal{F}\left(y, u_{2}\right), \\
u_{2}^{\prime} & =\mathcal{G}\left(y, u_{2}\right) .
\end{aligned}
$$

Since

$$
\begin{aligned}
& s_{1}^{\prime \prime}=\frac{1}{2} \frac{a^{\prime}}{u_{2}-a} s_{1}^{\prime}-\frac{u_{2}}{u_{2}-a} s_{1}, \\
& s_{2}^{\prime \prime}=\frac{1}{2} \frac{a^{\prime}}{u_{2}-a} s_{2}^{\prime}-\frac{u_{2}}{u_{2}-a} s_{2},
\end{aligned}
$$


the system (29) becomes

$$
\begin{aligned}
& \left(u_{2}-a\right) u_{1}^{\prime \prime}-\frac{1}{2} a^{\prime} u_{1}+u_{2} u_{1}=\mathcal{F}\left(y, u_{2}\right), \\
& u_{2}^{\prime}=\mathcal{G}\left(y, u_{2}\right) .
\end{aligned}
$$

The two remaining symmetries required to specify the system come from the particular solution of (26a) and its consequence in (25b). The particular solution of (26a) follows from that of the original equation (23) and the use of Laplace's method of variation of parameters. The symmetries can be written as

$$
\begin{aligned}
& \Sigma_{3}=A_{0} \partial_{y}+C_{0} u_{1} \partial_{u_{1}}+\partial_{u_{2}}, \\
& \Sigma_{4}=A_{0} u_{2} \partial_{y}+\left(C_{0} u_{2}+\gamma_{0}\right) u_{1} \partial_{u_{1}}+u_{2} \partial_{u_{2}}=u_{2} \Sigma_{3}+\gamma_{0} u_{1} \partial_{u_{2}},
\end{aligned}
$$

where $\gamma_{0}$ is a constant, $C_{0}$ is given by $(25 \mathrm{~b})$ and

$$
\begin{aligned}
A_{0}= & \frac{1}{4 W}\left\{\sigma_{1}^{2} \int \sigma_{2}^{2}\left[\frac{a^{\prime \prime}}{\left(u_{2}-a\right)^{2}}+\frac{a^{\prime 2}}{\left(u_{2}-a\right)^{3}}+\frac{4 a}{\left(u_{2}-a\right)^{2}}\right] \mathrm{d} y\right. \\
& -\sigma_{1} \sigma_{2} \int \sigma_{1} \sigma_{2}\left[\frac{a^{\prime \prime}}{\left(u_{2}-a\right)^{2}}+\frac{a^{\prime 2}}{\left(u_{2}-a\right)^{3}}+\frac{4 a}{\left(u_{2}-a\right)^{2}}\right] \mathrm{d} y \\
& \left.+\sigma_{2}^{2} \int \sigma_{1}^{2}\left[\frac{a^{\prime \prime}}{\left(u_{2}-a\right)^{2}}+\frac{a^{\prime 2}}{\left(u_{2}-a\right)^{3}}+\frac{4 a}{\left(u_{2}-a\right)^{2}}\right] \mathrm{d} y\right\} .
\end{aligned}
$$

In fact the complexity of the expression for the symmetry is not of importance. Rather it is the structure found in $\Sigma_{3}$ and $\Sigma_{4}$.

A symmetry of the original system (9) has the general form

$$
\Gamma=\tau \partial_{t}+\xi \partial_{x}+\eta \partial_{y}
$$

in which the variable dependence in the coefficient functions $\tau, \xi$ and $\eta$ is not specified since for the symmetries of interest to us this is determined through the connection to the point symmetries listed in (24). We follow the line of transformations through conversion to polar, i.e. $x=r \cos \theta, y=r \sin \theta$ and

$$
\Gamma \longrightarrow \tau \partial_{t}+(\xi \cos \theta+\eta \sin \theta) \partial_{r}+\frac{1}{r}(-\xi \sin \theta+\eta \cos \theta) \partial_{\theta}
$$

which for compactness of notation we write as

$$
\Gamma \longrightarrow \tau \partial_{t}+R \partial_{r}+\Theta \partial_{\theta},
$$

expression as the system of four first-order differential equations, i.e. $r=w_{1}, \theta=w_{2}, \dot{r}=w_{3}$, $\dot{\theta}=w_{4}$ and

$$
\Gamma \longrightarrow \tau \partial_{t}+R \partial_{w_{1}}+\Theta \partial_{w_{2}}+(\dot{R}-\dot{r} \dot{\tau}) \partial_{w_{3}}+(\dot{\Theta}-\dot{\theta} \dot{\tau}) \partial_{w_{4}}
$$

the reduction of order which means the elimination of $t$ and the use of $w_{2}=y$ as the independent variable giving the operator

$$
\Gamma \longrightarrow R \partial_{w_{1}}+\Theta \partial_{y}+(\dot{R}-\dot{r} \dot{\tau}) \partial_{w_{3}}+(\dot{\Theta}-\dot{\theta} \dot{\tau}) \partial_{w_{4}}
$$

Finally we have the elimination of $w_{3}$ and the introduction of the two new dependent variables $u_{1}=1 / w_{1}$ and $u_{2}=w_{1}^{4} w_{4}^{2}-a(y)$ so that

$$
\Gamma \longrightarrow\left(\Theta-(\dot{\Theta}-\dot{\theta} \dot{\tau}) a^{\prime}\right) \partial_{y}-u_{1}^{2} R \partial_{u_{1}}+\left(4 w_{1}^{3} w_{4}^{2} R+2 w_{1}^{4} w_{4}(\dot{\Theta}-\dot{\theta} \dot{\tau})\right) \partial_{u_{2}} .
$$


We are now in a position to express the symmetries (27) in terms of the original variables of our toy Ermakov system. After a modest amount of manipulation we obtain the symmetries

$$
\begin{aligned}
\Delta_{1}= & \left(\int s_{1}(\theta) r \mathrm{~d} t\right) \partial_{t}+s_{1}(\theta)\left(x \partial_{x}+y \partial_{y}\right) \\
\Delta_{2}= & \left(\int s_{2}(\theta) r \mathrm{~d} t\right) \partial_{t}+s_{2}(\theta)\left(x \partial_{x}+y \partial_{y}\right) \\
\Delta_{3}= & \left\{\int\left[\frac{\dot{\Theta}}{\dot{\theta}}-\frac{1}{2 r^{4} \dot{\theta}^{2}}-2 C_{0}\right] \mathrm{d} t\right\} \partial_{t}-\left[C_{0} x+y\left(A_{0}+\frac{\dot{a}}{2(x \dot{y}-\dot{x} y)^{2}}+2 C_{0} \dot{a}\right)\right] \partial_{x} \\
& +\left[-C_{0} y+x\left(A_{0}+\frac{\dot{a}}{2(x \dot{y}-\dot{x} y)^{2}}+2 C_{0} \dot{a}\right)\right] \partial_{y}, \\
\Delta_{4}= & \left((x \dot{y}-\dot{x} y)^{2}+a\right) \Delta_{3}+\frac{\gamma}{x^{2}+y^{2}}\left(x \partial_{x}+y \partial_{y}\right), \\
\Delta_{5}= & \partial_{t},
\end{aligned}
$$

where the fifth symmetry is introduced as the symmetry responsible for the reduction of order by the change of independent variable from $t$ to $\theta$ and $s_{1}$ and $s_{2}$ are linearly independent solutions of (23).

In (30) we have a representation of the complete symmetry group of our original system (9). The algebra of the symmetries may be written as $A_{1} \oplus\left\{A_{2} \oplus_{s} 2 A_{1}\right\}$ in the notation of the Mubarakzyanov classification scheme $[31,34,35,36]$. The first Abelian subalgebra comprises $\Delta_{5}$ and the second abelian subalgebra the solution symmetries $\Delta_{1}$ and $\Delta_{2}$.

Note that the four symmetries coming from the system (22), (23) are nonlocal only in the coefficient of $\partial_{t}$. The symmetries $\Delta_{1}$ and $\Delta_{2}$ are point in the spatial variables as is the case with the nonlocal symmetries used by Krause to specify the Kepler Problem. The symmetries $\Delta_{3}$ and $\Delta_{4}$ are generalised in the spatial variables. Given that the original system is autonomous, the concentration of the nonlocality in the coefficient of $\partial_{t}$ is not surprising since the integral does not enter of itself into the action of the symmetry on the system.

\section{Conclusion}

In this paper we have considered a very simple example of the class of problems known as Ermakov systems. The simplicity of the choice was deliberate for we were then able to concentrate upon the important concepts rather than becoming enmired in distracting manipulations. Even still the conversion of the symmetries from their simple representations for the system (22) and (23) to those given in (30) was not a completely trivial exercise. We have seen that a representation of the complete symmetry group of this toy Ermakov system cannot be achieved by means of Lie point symmetries. It is necessary to look to nonlocal symmetries. Nevertheless our toy system belongs to the class of superintegrable problems. It is also of some interest that by means of the method of reduction of order we were able to reduce the Ermakov system, fourth-order and nonlinear, to a third-order system of linear equations. This same reduction has been observed for other problems, but there seem to be some essential differences due to the geometric implications of the transformation of the independent variable.

As a final remark we recall that the coupled system (9) is superintegrable. Other superintegrable systems include the simple harmonic oscillator and the Kepler Problem. The former enjoys a richness of symmetry which is not lessened by the adjoining of a further dimension

or two. The latter never has a sufficient number of Lie point symmetries for its complete specification be the dimension two or twenty-two. Yet it is the classic example of a natural superintegrable system. The Ermakov system considered here occupies a middle position in that 
it has sufficient Lie point symmetries as a scalar system to be more than superintegrable and yet does not have the requisite number of point symmetries to be completely specifiable. Here we have detailed a strategy to reconcile the superintegrablity of the system and its ease of complete specification. The strategies for the resolution of particular problems may vary, but they have the same underlying basis which is to seek that representation in which the superintegrability is supported by an equally generous supply of Lie point symmetries for they are the class of symmetry with which to work is the easiest.

The conventional representations of systems such as the cojoined Ermakov system considered here are not necessarily those for which the representation of the Lie algebraic properties are the simplest.

\section{Acknowledgements}

PGLL thanks the University of KwaZulu-Natal for its continuing support.

[1] Andriopoulos K., Leach P.G.L., Flessas G.P., Complete symmetry groups of ordinary differential equations and their integrals: some basic considerations, J. Math. Anal. Appl., 2001, V.262, 256-273.

[2] Andriopoulos K., Leach P.G.L., The economy of complete symmetry groups for linear higher-dimensional systems, J. Nonlinear Math. Phys., 2002, V.9, suppl. 2, 10-23.

[3] Andriopoulos K., Symmetries and complete symmetry groups of differential equations, Dissertation, Department of Mathematics, University of the Aegean, Karlovassi 83200, Greece, 2002.

[4] Andriopoulos K., Leach P.G.L., Transitivity and intransitivity of complete symmetry groups, Preprint, Department of Information and Communication Systems Engineering, University of the Aegean, Karlovassi 83200, Greece, 2005.

[5] Andriopoulos K., Leach P.G.L., Wavefunctions for the time-dependent linear oscillator and Lie point symmetries, J. Phys. A: Math. Gen., 2005, V.38, 4365-4374.

[6] Burgan J.-R., Sur les groupes de transformation en physique mathématique. Application aux fluides de l'éspace des phases et à la mécanique quantique, Thèse, Université d'Orléans, Orléans, France, 1978.

[7] Burgan J.-R., Feix M.R., Fijalkow E., Gutierrez J., Munier A., Utilisation des groupes de transformation pour la resolution des équations aux derivées partielles, in Applied Inverse Problems, Editor P.C. Sabatier, Cahiers Mathématiques, Montpellier, 1978, 67-76.

[8] Eliezer C.J., Gray A., A note on the time-dependent harmonic oscillator, SIAM J. Appl. Math., 1976, V.30, 463-468.

[9] Ermakov V., Second-order differential equations. Conditions of complete integrability, Universitetskie Izvestiya, Kiev, 1880, N 9, 1-25 (translated by A.O. Harin).

[10] Govinder K.S., Leach P.G.L., Generalized Ermakov systems in terms of $s l(2, \mathbb{R})$ invariants, Qucst. Math., 1993, V.16, 405-412.

[11] Govinder K.S., Leach P.G.L., Algebraic properties of angular momentum type first integrals, Lie Groups Appl., 1994, V.1, 95-102.

[12] Günther N.J., Leach P.G.L., Generalized invariants for the time-dependent harmonic oscillator, J. Math. Phys., 1977, V.18, 572-576.

[13] Haas F., Goedert J., On the Hamiltonian structure of Ermakov systems, J. Phys. A: Math. Gen., 1996, V.29, 4083-4092.

[14] Haas F., Goedert J., Dynamical symmetries and the Ermakov invariant, Phys. Lett. A, 2001, V.279, 181-188.

[15] Haas F., Goedert J., Lie point symmetries for reduced Ermakov systems, Phys. Lett. A, 2004, V.332, 25-34.

[16] Karasu (Kalkanlı) A., Leach P.G.L., Nonlocal symmetries and integrable systems, J. Nonlinear Math. Phys., 2005, submitted.

[17] Krause J., On the complete symmetry group of the classical Kepler system, J. Math. Phys., 1994, V.35, $5734-5748$.

[18] Krause J., On the complete symmetry group of the Kepler problem, in Proceedings of the XXth International Colloquium on Group Theoretical Methods in Physics, Editor A. Arima, Singapore, World Scientific, 1995, 286-290. 
[19] Kruskal M., Asymptotic theory of Hamiltonian and other systems with all solutions nearly periodic, J. Math. Phys., 1962, V.3, 806-828.

[20] Leach P.G.L., On a direct method for the determination of an exact invariant for the time-dependent harmonic oscillator, J. Austral. Math. Soc. Ser. B, 1977, V.20, 97-105.

[21] Leach P.G.L., Applications of the Lie theory of extended groups in Hamiltonian mechanics: the oscillator and the Kepler problem, J. Austral. Math. Soc. Ser. B, 1980, V.23, 173-186.

[22] Leach P.G.L., Mahomed F.M., Maximal subalgebra associated with a first integral of a system possessing $\operatorname{sl}(3, \mathbb{R})$ symmetry, J. Math. Phys., 1988, V.29, 1807-1813.

[23] Leach P.G.L., Generalized Ermakov systems, Phys. Lett. A, 1991, V.158, 102-106.

[24] Leach P.G.L., Nucci M.C., Reduction of the classical MICZ-Kepler problem to a two-dimensional linear isotropic harmonic oscillator, J. Math. Phys., 2004, V.45, 3590-3604.

[25] Lewis H.R.Jr., Classical and quantum systems with time-dependent harmonic oscillator-type Hamiltonians, Phys. Rev. Lett., 1967, V.18, 510-512.

[26] Lewis H.R.Jr., Motion of a time-dependent harmonic oscillator and of a charged particle in a time-dependent, axially symmetric, electromagnetic field, Phys. Rev., 1968, V.172, 1313-1315.

[27] Lewis H.R.Jr., Class of exact invariants for classical and quantum time-dependent harmonic oscillators, J. Math. Phys., 1968, V.9, 1976-1986.

[28] Lewis H.R.Jr., Riesenfeld W.B., An exact quantum theory of the time-dependent harmonic oscillator and of a charged particle in a time-dependent electromagnetic field, J. Math. Phys., 1969, V.10, 1458-1473.

[29] Lewis H.R., Leach P.G.L., A direct approach to finding exact invariants for one-dimensional time-dependent classical Hamiltonians, J. Math. Phys., 1982, V.23, 2371-2374.

[30] Mahomed F.M., Leach P.G.L., Symmetry Lie algebras of $n$th order ordinary differential equations, J. Math. Anal. Appl., 1990, V.151, 80-107.

[31] Morozov V.V., Classification of six-dimensional nilpotent Lie algebras, Izv. Vys. Uchebn. Zaved. Matematika, 1958, N 4 (5), 161-171.

[32] Moyo S., Leach P.G.L., Exceptional properties of second and third order ordinary differential equations of maximal symmetry, J. Math. Anal. Appl., 2000, V.252, 840-863.

[33] Moyo S., Leach P.G.L., A note on the construction of the Ermakov-Lewis invariant, J. Phys. A: Math. Gen., 2002, V.35, 5333-5345.

[34] Mubarakzyanov G.M., On solvable Lie algebras, Izv. Vys. Uchebn. Zaved. Matematika, 1963, N 1 (32), $114-123$.

[35] Mubarakzyanov G.M., Classification of real structures of five-dimensional Lie algebras, Izv. Vys. Uchebn. Zaved. Matematika, 1963, N 3 (34), 99-106.

[36] Mubarakzyanov G.M., Classification of solvable Lie algebras of sixth order with a non-nilpotent basis element, Izv. Vys. Uchebn. Zaved. Matematika, 1963, N 4 (35), 104-116.

[37] Notices of the American Mathematical Society, 2002, V.49, 490.

[38] Nucci M.C., The complete Kepler group can be derived by Lie group analysis, J. Math. Phys., 1996, V.37, $1772-1775$.

[39] Nucci M.C., Leach P.G.L., The determination of nonlocal symmetries by the method of reduction of order, J. Math. Anal. Appl., 2000, V.251, 871-884.

[40] Nucci M.C., Leach P.G.L., The harmony in the Kepler and related problems, J. Math. Phys., 2001, V.42, 746-764.

[41] Nucci M.C., Leach P.G.L., Jacobi's last multiplier and the complete symmetry group of the Euler-Poinsot system, J. Nonlinear Math. Phys., 2002, V.9, suppl. 2, 110-121.

[42] Nucci M.C., Leach P.G.L., Jacobi's last multiplier and symmetries for the Kepler Problem plus a lineal story, J. Phys. A: Math. Gen., 2004, V. 37, 7743-7753.

[43] Nucci M.C., Jacobi last multiplier and Lie symmetries: a novel application of an old relationship, J. Nonlinear Math. Phys., 2005, V.12, 284-304.

[44] Nucci M.C., Leach P.G.L., Jacobi's last multiplier and the complete symmetry group of the Ermakov-Pinney equation, J. Nonlinear Math. Phys., 2005, V.12, 305-320.

[45] Pillay T., Leach P.G.L., Chaos, integrability and symmetry, South African J. Sci., 2000, V.96, 371-376.

[46] Pinney E., The nonlinear differential equation $y^{\prime \prime}(x)+p(x) y+c y^{-3}=0$, Proc. Amer. Math. Soc., 1950, V.1, 681. 
[47] Ray J.R., Nonlinear superposition law for generalized Ermakov systems, Phys. Lett. A, 1980, V.78, 4-6.

[48] Ray J.R., Reid J.L., Noether's theorem and Ermakov systems for nonlinear equations of motion, Nuovo Cimento A (11), 1980, V.59, 134-140.

[49] Ray J.R., Reid J.L., Ermakov systems, nonlinear superposition principles and solutions of nonlinear equations of motion, J. Math. Phys., 1980, V.22, 91-95.

[50] Türkmen F., Leach P.G.L., Lie point symmetries of second-order ordinary differential equations, Preprint, School of Mathematical Sciences, Howard College, University of KwaZulu-Natal, Durban 4041, Republic of South Africa, 2005. 